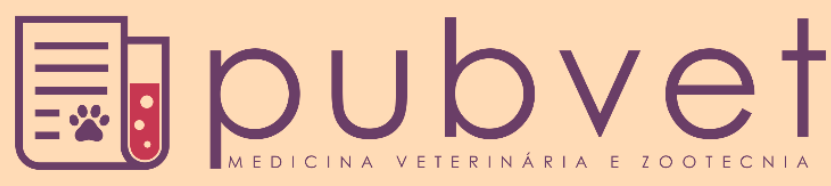

https://doi.org/10.31533/pubvet.v15n02a741.1-5

\title{
Manejo nutricional de cães e gatos domiciliados em São Luís - Maranhão
}

\author{
Sérgio Henrique Costa Júnior ${ }^{1 *} \bullet$, Daniel Welligton Brito de $\operatorname{Souza}^{2} \bullet$, Mikaelle Cristina Costa de \\ Souza $^{2}{ }^{\circ}$, Kamylla Martins Correia ${ }^{2}$, Leandra Patrícia da Silva Almeida ${ }^{2}$, Leandro Henrique \\ Veiga de Sousa ${ }^{1} \bullet$, Victória Torquato Fernandes dos $\operatorname{Santos}^{1}{ }^{\ominus}$, Larissa Fernanda Soares Lima ${ }^{1} \bullet$ \\ ${ }^{I^{*}}$ Mestrandos em Ciência Animal, Universidade Estadual do Maranhão, São Luís - MA, Brasil. \\ ${ }^{2}$ Acadêmicos em Medicina Veterinária, Universidade Estadual do Maranhão, São Luís - MA, Brasil. \\ *Autor para correspondência, E-mail: sergiocosta94@outlook.com
}

Resumo. O objetivo do estudo consistiu na construção do perfil do manejo nutricional de animais de companhia da cidade de São Luís - Maranhão através da aplicação de questionário composto por questões objetivas e discursivas em 107 pessoas domiciliadas em 38 bairros do município. Foram avaliados o grau de conhecimento sobre a alimentação animal, hábitos na oferta de comida aos seus pets e nível socioeconômico. O principal alimento oferecido aos animais foi a ração comercial seca, com gasto médio mensal de 148 reais na assistência nutricional, no entanto ainda há tutores que dão alimentação humana diariamente sem conhecimento da segurança e qualidade dos nutrientes no alimento oferecido. A fonte de informação mais citada consistiu na consulta veterinária de rotina e a internet. A maioria dos tutores relatam obedecer às exigências nutricionais mínimas, porém foi percebido uma variabilidade de critérios. Os tutores demonstraram conhecimento relativo ao manejo nutricional básico e preconiza-se uma maior disseminação técnica acerca desse tema visto que é vital para a manutenção da saúde dos cães e gatos.

Palavras-chave: alimentação animal, nutrição, pets, tutor

\section{Nutritional profile of household dogs and cats in São Luís - Maranhão}

\begin{abstract}
The objective of the study was to build the profile of the nutritional management of pets in the city of São Luís - Maranhão through the application of a questionnaire composed of objective and discursive questions in 107 people living in 38 neighborhoods in the city. The degree of knowledge about animal feeding, habits in the supply of food to their pets and socioeconomic level were evaluated. The main food offered to the animals was dry commercial food, with an average monthly expenditure of 148 reais on nutritional assistance, however there are still tutors who give human food daily without knowledge of the safety and quality of nutrients in the food offered. The most cited source of information was the routine veterinary consultation and the internet. Most tutors report complying with the minimum nutritional requirements, but a variability of criteria was noted. The tutors demonstrated knowledge related to basic nutritional management and a greater technical dissemination on this topic is recommended, since it is vital for maintaining the health of dogs and cats.
\end{abstract}

Keywords: animal feed, nutrition, pets, tutor

\section{Perfil nutricional de perros y gatos del hogar en São Luís - Maranhão}

Resumen. El objetivo del estudio fue construir el perfil del manejo nutricional de mascotas en la ciudad de São Luís - Maranhão a través de la aplicación de un cuestionario compuesto por preguntas objetivas y discursivas en 107 personas que viven en 38 barrios de la ciudad. 
Se evaluó el grado de conocimiento sobre alimentación animal, hábitos en el suministro de alimentos a sus mascotas y nivel socioeconómico. El principal alimento ofrecido a los animales fue el alimento comercial seco, con un gasto mensual promedio de 148 reales en asistencia nutricional, sin embargo, aún existen tutores que dan alimento humano diariamente sin conocimiento de la seguridad y calidad de los nutrientes en los alimentos ofrecidos. La fuente de información más citada fue la consulta veterinaria de rutina e Internet. La mayoría de los tutores informan cumplir con los requisitos nutricionales mínimos, pero se observó una variabilidad de criterios. Los tutores demostraron conocimientos relacionados con el manejo nutricional básico y se recomienda una mayor diseminación técnica sobre este tema, ya que es vital para el mantenimiento de la salud de perros y gatos.

Palabras clave: alimentación animal, nutrición, mascotas, tutor

\section{Introdução}

O perfil da nutrição de cães e gatos sofreu alterações nas últimas décadas. Anteriormente os restos alimentares de refeições destinadas ao consumo humano representavam a principal fonte de obtenção nutricional.

O mercado pet tem chamado a atenção devido seu crescimento exponencial nos últimos anos e marcado, sobretudo, pelo incremento na indústria de alimentação animal (ABINPET, 2019; Bermudes, 2016). O Brasil se destaca no mundo como um dos maiores produtores de alimentos para animais de companhia, existem aproximadamente 500 marcas e 85 fabricantes da indústria de alimentação para pet de acordo com Carciofi et al. (2009). Nesta situação, a nutrição é um dos principais fatores relacionados com a manutenção da saúde de cães e gatos, conforme citam Aptekmann et al. (2014) as práticas de alimentação realizadas para animais domiciliados são influenciadas pelas preferências e atitudes dos tutores.

A ausência do cuidado adequado com o manejo nutricional de cães e gatos propicia o surgimento de doenças nutricionais, como a obesidade, causada pelo excesso de gorduras, e sobrecarga da função renal, por exemplo (Fazenda, 2010; Oliveira et al., 2010). Entretanto, a partir de avanços na nutrição de animais de companhia, observa-se a comercialização de dietas comerciais com formulações cada vez mais sofisticadas e específicas, promovendo bem estar, melhora da saúde e redução do risco de doenças (ABINPET, 2019; Bermudes, 2016).

A formulação da ração, processamento, embalagem, armazenamento devem ser adequados às necessidades para cada estágio de vida do animal influenciado pela condição fisiológica, estilo de vida, enfermidades adquiridas e/ou congênitas têm sido objeto de estudo na Medicina Veterinária (Carciofi \& Jeremias, 2010).

A coleta de informações dos tutores de cães e gatos possibilita uma avaliação minuciosa e elucidação de problemas acerca da nutrição e promovendo ações para melhorar a qualidade de vida destes animais. Dessa forma, o objetivo do estudo consistiu na construção do perfil do manejo nutricional de animais de companhia da cidade de São Luís - Maranhão.

\section{Material e métodos}

Foram entrevistadas 107 pessoas domiciliadas em 38 bairros do município de São Luís - MA aplicando-se um questionário semiestruturado composto por questões objetivas e discursivas para obtenção do perfil de manejo alimentar implantado pelos tutores de cães e gatos, sem distinção de raça, idade e espécie.

A pesquisa foi realizada por meio de entrevistas entre os meses de março a maio de 2019 com esclarecimento e autorização dos tutores que foram escolhidos ao acaso, de acordo com a disponibilidade, para responder sobre seu grau de conhecimento sobre a alimentação animal, hábitos na oferta de comida aos seus pets e nível socioeconômico.

Os dados obtidos foram transferidos para o software Excel 2013, da Microsoft, e foram realizadas análises estatísticas (média, desvio padrão e porcentagem) a partir das frequências das informações coletadas. 


\section{Resultados e discussão}

A partir dos dados obtidos dos tutores, o quantitativo de pets consistiu em 121 cães e 49 gatos com idade média de 3,86 $\pm 3,2$ e 1,78 $\pm 2,01$ anos, respectivamente, com peso médio de 11,21 $\pm 10,1 \mathrm{~kg}$.

Em relação a renda média dos ludovicenses foi obtido o valor, em reais, de 3.707,40, de acordo com o censo de 2016 do IBGE. A renda média da população de São Luís consiste em 2.994,00 reais, dado semelhante ao encontrado no presente estudo. Animais de companhia necessitam de cuidados diários na higiene, imunidade e alimentação, por exemplo. Dessa forma, foi questionado o gasto médio na alimentação com cães e gatos obtendo-se o valor de 148 reais mensais. O que não corrobora com as análises de Aptekmann et al. (2014), onde 14\% e 15\% dos entrevistados classificaram a alimentação como um alto custo mensal de cães e gatos, respectivamente.

Sobre os tipos de alimentos que são oferecidos aos animais, a ração seca comercial foi a opção mais escolhida (57\%) entre os tutores, Lopes et al. (2019) observaram que 59,4\% os tutores de cães e gatos na cidade de Maceió também oferecem ração. Sendo uma alternativa mais prática de alimento diário, o que explicam Aptekmann et al. (2014) no Estado do Espírito Santo onde 90\% dos proprietários ofereciam esta opção aos seus animais. Fonseca et al. (2017) analisando o perfil e investimentos no mercado veterinário notaram que as vendas em diferentes estabelecimentos são representadas em $50 \%$ na compra de ração, petiscos e prestação de serviço.

O oferecimento de comida caseira para pets apresentou um baixo percentual $(6 \%)$, isto se deve ao modo de preparo que diferencia da comida humana, tendo custo, rotina pessoal e preferência como fatores determinantes. No entanto, a combinação ração mais comida caseira para pets e ração mais comida para consumo humano apresentaram valores de $13 \%$ e $21 \%$, respectivamente.

É descrito na literatura que alguns ingredientes da comida humana são prejudiciais aos animais podendo afetar diversos sistemas orgânicos, no presente estudo apenas 3\% dos animais ingerem refeições para consumo humano, dado similar ao encontrado por Lopes et al. (2019) e divergente ao perfil obtido por Aptekmann et al. (2014), onde 74\% dos proprietários ofereciam comida caseira humana. A opção de ração úmida apresentou apenas 3\% dos entrevistados.

As rações terapêuticas são uma nova opção de alimentação para os animais, elas possuem propriedades fundamentais no tratamento de algumas enfermidades, são facilmente encontradas nas lojas veterinárias. Dada a importância do manejo alimentar para algumas doenças que induzem alterações metabólicas e funcionais específicas (Pantoja et al., 2018; Zaine et al., 2014), como cardiopatias, nefropatias e obesidade animal.

Quando questionados sobre a existência dessas rações, 61\% dos proprietários relataram que conhecem esta opção alimentar, $37 \%$ relataram que não conheciam e $2 \%$ não responderam. No entanto, apenas $8 \%$ dos tutores relataram utilizar rações terapêuticas como a única forma de alimentação aos animais, devido ao alto valor destes produtos o tutor tende a adquirir outras marcas, Lopes et al. (2019) entrevistaram 96 tutores e nenhum utilizava as rações terapêuticas.

Frutas e legumes podem ser oferecidos aos animais; porém, é necessário saber os riscos que cada alimento pode oferecer, determinadas frutas podem ser ingeridas sem as sementes, alguns vegetais promovem disfunção fisiológica, no presente estudo $60 \%$ dos animais comem frutas e legumes, $38 \%$ não possuem o hábito e $2 \%$ dos tutores não souberam responder

Quando questionados sobre a administração de suplementos nutricionais aos animais, $41 \%$ nunca utilizou algum suplemento para melhorar o estado nutricional em cães e gatos, resultado similar dos tutores que já utilizaram (39\%). No presente estudo $20 \%$ das pessoas não souberam responder, o que explica a falta de divulgação acerca da nutrição animal na cidade de São Luís.

Saad \& França (2010) explicam que os proprietários estão cada vez mais exigentes com a nutrição de seus animais e apesar da diversidade em alimentos, deve-se apontar as vantagens e desvantagens sob o ponto de vista nutricional e segurança alimentar.

Dessa forma, $70,1 \%$ dos tutores relatam que estão suprindo as necessidades nutricionais, enquanto 29,9\% admitem que não estão suprindo a nutrição básica de seus animais, mesmo que seja uma análise positiva, mas há subjetividade nas repostas, pois fatores intrínsecos podem passar despercebidos. 
Os principais nutrientes incluem as proteínas, gorduras, carboidratos, fibras, vitaminas e minerais necessários para mantença e otimização do desempenho dos animais de companhia (França et al., 2011).

As consultas veterinárias de rotina são necessárias para diagnóstico precoce de distúrbios nutricionais, o que justifica 56\% dos entrevistados afirmaram não possuir conhecimento da formulação dos alimentos industrializados para cães e gatos conforme Lopes et al. (2019).

Os meios mais utilizados em casos de dúvidas sobre alimentação animal foram: $40 \%$ consultas veterinárias, $31 \%$ Internet, $18 \%$ Petshops, $5 \%$ amigos e familiares, $1 \%$ revistas, $1 \%$ TV ou rádio e $5 \%$ não responderam, estes dados são similares aos obtidos de questionários aplicados em tutores nos Estados do Espírito Santo e Alagoas e nos Estados Unidos e Austrália (Aptekmann et al., 2014; Laflamme et al., 2008; Lopes et al., 2019), ressaltando a importância do papel do médico veterinário para um correto manejo nutricional, conforme demonstrado por Lopes et al. (2019) que 50\% dos proprietários afirmaram ter consultado um veterinário ou zootecnista.

O armazenamento da ração seca comercial requer uma proteção eficaz para manter a qualidade nutricional e palatabilidade, as embalagens das rações para alimentação animal devem ser de plástico, sendo seguras e resistentes para garantir a devida proteção ao produto (Kunz et al., 2005).

A compra de ração a granel é uma prática antiga e até hoje muito utilizada, possibilita a aquisição de pequenas quantidades de ração, quando questionados, $58 \%$ dos tutores compram a granel e $38 \%$ não compram, 4\% não souberam responder. Lopes et al. (2019) observaram que a compra da ração era influenciada pelo modo de armazenamento $(79,2 \%)$ e o prazo de validade $(80,2 \%)$ antes da aquisição. E, após a compra da ração, o local da ração mais citado pelos tutores no presente estudo foram: depósito plástico com tampa (61\%), embalagem original (21\%), saco plástico (10\%), lata (4\%) e sem resposta (4\%).

Quando questionados sobre a frequência da alimentação diária dos animais, os tutores relataram que oferecem alimentação duas vezes ao dia (52\%), três vezes ao dia (24\%), uma vez ao dia (13\%), quatro vezes ao dia (5\%), cinco vezes ao dia (3\%) e 3\% não souberam responder. Laflamme et al. (2008) observaram que gatos tinham alimento disponível durante todo o dia e os cães alimentados duas vezes ao dia, porém afirmam Aptekmann et al. (2014) que existe diferença na frequência na oferta de alimentos aos animais.

O critério para quantificar o oferecimento de ração diária é determinante para o grau nutricional e escore corporal dos animais, no questionário os tutores responderam seus critérios pessoais. A satisfação do animal foi a mais citada, seguido da opção de não ter um critério para alimentação diária. Apenas oito tutores relatam que obedecem às informações da embalagem/rótulo e sete tutores, sob orientação do Médico Veterinário. Critérios como peso do animal, comportamento animal, quantificação por copos de plástico, idade, tamanho do comedouro também foram citados no presente estudo. No entanto, a maioria dos tutores $(n=28)$ não souberam citar seu critério.

Passear com os animais consiste num hábito saudável que reduz o estresse e evita obesidade, no entanto, 29\% dos tutores não tem o costume de levar seus animais para passear em locais públicos e $8 \%$ não responderam. $63 \%$ dos tutores possuem este hábito e quando questionados sobre a frequência de passeio responderam: uma, duas, três e quatro por semana, apenas aos finais de semana e todos os dias, foi obtido $18 \%, 24 \%, 14 \%, 16 \%, 16 \%, 12 \%$, respectivamente.

\section{Conclusão}

Pode-se inferir que os tutores de animais de companhia de São Luís possuem certo conhecimento relativo ao manejo nutricional básico. No entanto, preconiza-se uma maior difusão técnica acerca desse tema visto que é vital para a manutenção da saúde dos cães e gatos.

\section{Referências}

ABINPET. (2019). Caderno especial Abinpet-Associação Brasileira da Indústria de Produtos para Animais de Estimação. Agro Analysis, 35(1), 35-40.

Aptekmann, K. P., Suhett, W. G., Junior, A. F. M., Souza, G. B., Tristão, A. P. P. A., Adams, F. K., Aoki, C. G., Junior, R. J. G. P., Carciofi, A. C., \& Tinucci-Costa, M. (2014). Aspectos nutricionais 
e ambientais da obesidade canina. Ciência Rural, 44(11), 2039-2044. DOI: https://doi.org/10.1590/0103-8478cr20130524

Bermudes, P. (2016). Tendências de mercado \& perfil do consumidor. In XV Congresso sobre Nutrição de Animais de Estimação CBNA PET. Colégio Brasileiro de Nutrição Animal.

Carciofi, A. C., \& Jeremias, J. T. (2010). Progresso científico sobre nutrição de animais de companhia na primeira década do século XXI. Revista Brasileira de Zootecnia, 39, 35-41. DOI: https://doi.org/10.1590/s1516-35982010001300005

Carciofi, A. C., Teshima, E., Bazolli, R. S., Brunetto, M. A., Vasconcellos, R. S., de Oliveira, L. D., \& Pereira, G. T. (2009). Qualidade e digestibilidade de alimentos comerciais de diferentes segmentos de mercado para cães adultos. Revista Brasileira de Saúde e Produção Animal, 10(2), 489-500.

Fazenda, M. I. N. (2010). Estudo da relação entre a obesidade e a hipertensão em cães. In Faculdade de Medicina Veterinária: Vol. Master of. Universidade Técnica de Lisboa.

Fonseca, G. O., Hespanhol, R. M., \& Pereira, D. R. (2017). Análise mercadológica do segmento pet: estudo de caso utilizando aprendizado de máquina. Revista Latino-Americana de Inovação $e$ Engenharia de Produção, 5(8), 117-135. DOI: https://doi.org/10.5380/relainep.v5i8.56495

França, J., Saad, F., Saad, C. E. P., Silva, R. C., \& Reis, J. S. (2011). Avaliação de ingredientes convencionais e alternativos em rações de cães e gatos. Revista Brasileira de Zootecnia, 40, 222-231.

Kunz, A., Chiochetta, O., Miele, M., Girotto, A. F., \& Sangoi, V. (2005). Comparativo de custos de implantação de diferentes tecnologias de armazenagem/tratamento e distribuição de dejetos de suínos. Embrapa Suínos e Aves, 42, 1-16.

Laflamme, D. P., Abood, S. K., Fascetti, A. J., Fleeman, L. M., Freeman, L. M., Michel, K. E., Bauer, C., Kemp, B. L. E., Doren, J. R. Van, \& Willoughby, K. N. (2008). Pet feeding practices of dog and cat owners in the United States and Australia. Journal of the American Veterinary Medical Association, 232(5), 687-694. DOI: https://doi.org/10.2460/javma.232.5.687

Lopes, L. A., Lira, R. C., Camargo, K. S., \& Santos, E. L. (2019). Manejo nutricional de cães e gatos domiciliados no município de Maceió, Alagoas, Brasil. Revista de Educação Continuada Em Medicina Veterinária e Zootecnia Do CRMV-SP, 17(3), 36-40. DOI: https://doi.org/10.36440/recmvz.v17i3.38002

Oliveira, M. C., Nascimento, B. C. L., \& Amaral, R. W. C. (2010). Obesidade em cães e seus efeitos em biomarcadores sanguíneos-revisão de literatura. PUBVET, 4, Art. 795-801.

Pantoja, J. C., Cabral, Í. S., Farias, T. S., Amaral, T. E. S., \& Barbosa, C. R. (2018). Alimentação de cães $\mathrm{e}$ gatos cardiopatas. PUBVET, 12(11), 1-8. DOI: https://doi.org/10.31533/pubvet.v12n11a213.1-8

Saad, F. M. O. B., \& França, J. (2010). Alimentação natural para cães e gatos. Revista Brasileira de Zootecnia, 39(1), 52-59. DOI: https://doi.org/10.1590/s1516-35982010001300007

Zaine, L., Monti, M., Vasconcellos, R. S., \& Carciofi, A. C. (2014). Nutracêuticos imunomoduladores com potencial uso clínico para cães e gatos. Semina: Ciências Agrárias, 35(4), 2513-2529. DOI: https://doi.org/10.5433/1679-0359.2014v35n4suplp2513.

\section{Histórico:}

Recebido: 12 de agosto, 2020. Aprovado: 19 de setembro, 2020.

Disponível online: 12 de dezembro, 2020.
Licenciamento: Este artigo é publicado na modalidade Acesso Aberto sob a licença Creative Commons Atribuição 4.0 (CC-BY 4.0), a qual permite uso irrestrito, distribuição, reprodução em qualquer meio, desde que $o$ autor e a fonte sejam devidamente creditados. 